\title{
Parallel Image Matching Based on Local Entropy Difference Vector
}

\author{
Kai-Kun Dong, Le-Jun Chi, Li Guo \\ dkk@hitwh.edu.cn \\ School of Computer Science and Technology, Harbin Institute of Technology at Weihai, \\ Weihai, 264209, P.R.China
}

\begin{abstract}
This paper presents a novel parallel image matching algorithm based on the local entropy difference vector (LEDV). Spatial information is introduced into the definition of image entropy and a thresholding operation is employed to limit the contribution of a mismatching block to the matching result. The proposed approach confirms the robustness of the LEDV to irradiation distortion, geometric distortion, local noise interference and occlusion, coupled with the transform of image entropy definition to make it suitable for parallel processing, can lead to good matching result, which is validated by experiments on a variety of images in natural environment.
\end{abstract}

Keywords: image processing, image matching, local entropy difference vector, SIMD

\section{Introduction}

Image matching is an inarguably important operation in computer vision and image processing. It has been widely used in aircraft navigation, resource analysis, weather forecast and medical diagnosis [1]. Image matching algorithms proposed in the past 30 years can be classified into two types: template matching methods based on color or grey correlation and feature matching skills based on image features extracted [2]. Compared with the former, the latter usually shows advantages of being robust to geometric distortion, gray difference and noise interference. However, most of them need to extract feature points or edge of the image, and the matching results depend mainly on the quality of feature extraction. In fact, it is still a difficult work to segment or extract feature from the image of scenery in natural environment [3].

In aircraft navigation system, aerial photographs or satellite images of the areas where the aircraft is going to fly over are taken and stored in computer memory on the aircraft as reference image before the flight. While flying, the aircraft takes photographs of the areas where it arrives, namely real-time image, and matches it with the reference image to determine the position of the aircraft.

In this paper, a new definition of image entropy is presented and a parallel image matching algorithm dedicated to a SIMD array computer is proposed.

The rest of the paper is organized as follows. Section 2 defines the LEDV. Section 3 introduces the DZJ-I array computer in brief. Section 4 describes the proposed algorithm. Section 5 illustrates the experimental results. Section6 draws conclusions.

\section{Local Entropy Difference Vector}

Since the concept of entropy in physics was introduced into information theory by Shannon, it has been applied in image

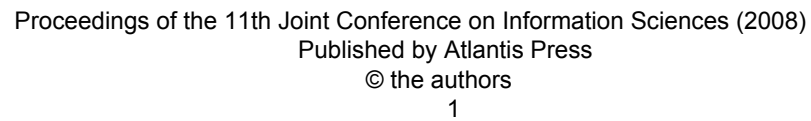


processing tasks, such as image segmentation, compression, retrieval, restoration, reconstruction, enhancement, correction, fusion and matching.

Let $F=[f(x, y)]_{M \times N}$ be an image of size $M \times N$, where $f(x, y)$ is the gray value of pixel $(x, y), 1 \leqslant x \leqslant M, 1 \leqslant y \leqslant N$, and $f(x$, $y) \in G_{L}=\{0,1, \ldots, L-1\}$, the set of gray levels. The entropy of $F$ is defined as

$$
H_{f}=-\sum_{i=1}^{M} \sum_{j=1}^{N}\left(p_{i j} \times \log p_{i j}\right)
$$

where

$$
p_{i j}=f(i, j) / \sum_{i}^{M} \sum_{j}^{N} f(i, j) \cdot
$$

$p_{i j}$ denotes the probability of $f(x, y)$. If $F$ is a subimage, $H_{f}$ is called local entropy.

The entropy gives additive property information of an image and it has been proved in reference [4] to be robust to irradiation distortion, geometric distortion and noise interference when being used as a kind of feature description for image matching. However, the definition has two important shortcomings. First, no spatial information of the image has been introduced. Which means two images entirely different in structure may have the same entropy. Second, from the viewpoint of parallel computing, the following computations must be performed in sequence for the reason of data dependence.

- $S=\sum_{i=1}^{M} \sum_{j=1}^{N} f(i, j)$

- $p_{i j}=f(i, j) / \mathrm{s}$

- $H_{f}=-\sum_{i=1}^{M} \sum_{j=1}^{N}\left(p_{i j} \times \log p_{i j}\right)$

Therefore, we define the entropy of an image as a two-dimensional vector. For image $F$, assume $m, n$ are two positive integers that go into $M, N$ respectively. Segment image $F$ into $(M / m) \times(N / n)$ blocks, and the size of each block is $m \times n$. Define the local entropy vector of image $F$ as $H_{F}$, where $H_{F}(p, q)=H_{f}(p, q)$, $p=0,1, \ldots, M / m-1, q=0,1, \ldots, N / n-1$, and $H_{f}(p, q)$ is the local entropy of block ( $p$, $q)$. To eliminate the data dependence and reduce computation, $H_{F}(p, q)$ is calculated as

$$
\begin{array}{r}
H_{F}(p, q)=-\frac{1}{S} \times \sum_{i=1}^{m} \sum_{j=1}^{n}(f(p \times m+i, q \times n+j) \\
\times \log f(p \times m+i, q \times n+j))+\log S,(2)
\end{array}
$$

where

$$
S=\sum_{i=1}^{m} \sum_{j=1}^{n} f(p \times m+i, q \times n+j) .
$$

It is easy to prove that equation (2) is equivalent to equation (1). Suppose $G$ is an image with the same size of $F$, then we have the local entropy absolute difference vector $D_{F, G}=H_{F}-H_{G}$, i.e.

$$
D_{F, G}(p, q)=\left|H_{F}(p, q)-H_{G}(p, q)\right| \text {. }
$$

To avoid the wrong matching result caused by severe local interference noises or being partly occluded, a threshold function is applied to $D_{F, G}$ in our algorithm, i.e.

$$
D_{F, G}(p, q)=\left\{\begin{array}{ll}
0 & \text { if } D_{F, G}(p, q) \leq T \\
1 & \text { if } D_{F, G}(p, q)>T
\end{array}\right. \text {. }
$$

Where $T$ is a threshold determined beforehand by building the histogram of components of the local entropy absolute difference vector between real-time images and the candidate matching images.

$D_{F, G}$ defined in (3) and (4) is called as the local entropy difference vector between $F$ and $G$.

\section{The DZJ-I Array Computer}

Image processing tasks in remote sensing and computer vision require an enormous amount of computation, especially in practical, real-time applications. Therefore, DZJ-I, a processor array aimed at low-and intermediate-level image processing operations is designed and implemented by the Parallel Computing Laboratory of Harbin Institute of Technology (HIT). Its architecture is shown in Fig. 1.

The essential feature of DZJ-I is its nonlinear storage schemes and compound multistage interconnection network which 
provide conflict-free access of rows, columns, diagonals, contiguous blocks and distributed blocks of a matrix $[5,6]$.

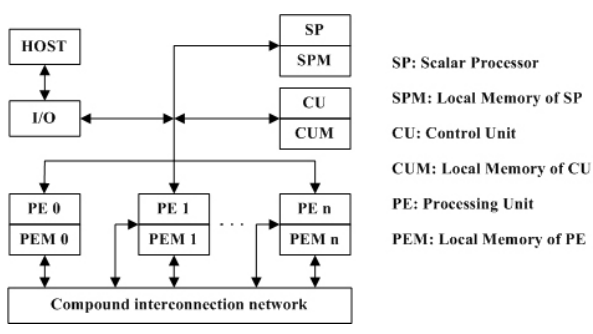

Fig. 1: Architecture of DZJ-I array computer.

\section{The Proposed Algorithm}

Based on the LEDV defined above, a parallel image matching algorithm on DZJ-I is put forward. Assuming the size of realtime image $F$ is $M \times N$, the number of PEs is $K$, and $(M / m) \times(N / n)$ can be divided by $K$, the proposed algorithm consists of following steps:

1. Segment $F$ into blocks of size
$m \times n$. Allocate image gray values
of block $(p, q)$ to the local memory
of $\mathrm{PE}_{i}$, where $i=((M / m) \times(N / n))$
$\bmod K, 0 \leq p \leq M / m-1$, and $0 \leq q \leq$
$N / n-1$.
2. For $p=0,1, \ldots, M / m-1, q=0,1, \ldots$,
$N / n-1$, calculate $H_{F}(p, q)$ by
equation $(2)$ on $\mathrm{PE}_{i}$, where $i=$
$(p \times m+q)$ mod $K$.
3. Select a subimage of the same size
with $F$ from reference image as
candidate matching image $F$ '.
Segment $F^{\prime}$ and allocate the image
gray values in the same way as $F$
in step1.
4. Calculate $H_{F}$, the local entropy
vector of $F^{\prime}$ in the same way as $F$
in step2.
5. Calculate the absolute difference
vector $D_{F, F}=H_{F}-H_{F}$, where
$D_{F, F}(p, q)=\left|H_{F}(p, q)-H_{F}(p, q)\right|$,

$0 \leq p \leq M / m-1$, and $0 \leq q \leq N / n-1$.

6. For $p=0,1, \ldots, M / m-1, q=0,1, \ldots$, $N / n-1$, if $D_{F, F}(p, q) \leq T$, then set the value of $D_{F, F},(p, q)$ with 0 ; otherwise set it with 1 .

7. Calculate the sum of all the components of $D_{F, F}$, and take it as the matching result between $F$ and $F$ '.

8. Select another candidate matching image that has not been matched and go to step3 until all the candidate matching images have been matched.

9. Take the candidate matching image which has the smallest matching result as the ultimate matching image.

Taylor expansion is often used in order to simplify the computation of entropy. But experiments carried out by us show that the approximate calculation error may lead to wrong matching in some cases. To solve the problem, a logarithmic table is stored in local memory of DZJ-I. The transform from (1) to (2) made it feasible and easy to implement because the gray levels of an image are limited. The algorithm is improved in both processing speed and accuracy.

\section{Experiment Results}

The proposed algorithm has been tested on a variety of aerial photographs and satellite images. To demonstrate the performance of the proposed algorithm, we compare the image matching results with other algorithms. An example is shown in Fig. 2. Fig. 2 (a) is a satellite reference image of size $256 \times 256$. Fig. 2 (b) is a real-time image of size $64 \times 64$ which has a 3 degree rotation and gray difference compared with Fig. 2 (a). Fig. 2 (c) is the matching result of gray correlation matching algorithm based on mean absolute differences (MAD). Fig. 2 (d) is the matching result of minimum entropy difference algorithm. 
Fig. 2 (e) is the matching result using the proposed algorithm. The comparisons illustrate that the proposed approach performs image matching task more effectively than those to be compared with under complex matching conditions in natural environment.

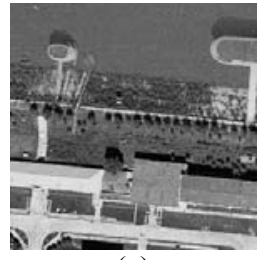

(a)

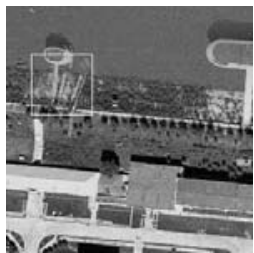

(d)

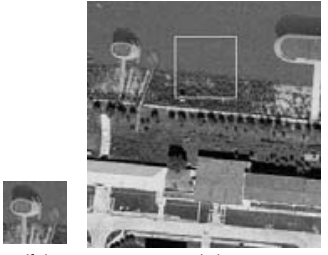

(c)

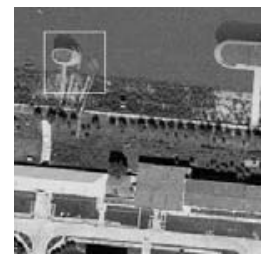

(e)
Fig. 2: (a) Reference image. (b) Real-time image. (c) The matching result of MAD algorithm. (d) The matching result of minimum entropy difference algorithm. (e) The matching result using the proposed algorithm.

\section{Conclusion}

The proposed algorithm based on the LEDV introduces spatial information into the definition of image entropy. By employing a thresholding operation, the contribution of a mismatching block to the matching result is limited to reciprocal of the blocks number. The transform from (1) to (2) made it possible to store a logarithmic table in the local memory and suitable for parallel processing and the processing is markedly sped up. Experiment results confirm that the proposed algorithm is robust to irradiation distortion, geometric distortion, local severe noise interference and occlusion.

\section{Acknowledgments}

This work was funded by the Natural Science Foundation of Shandong Province (2006ZRA10010), by the Science Foundation of HIT at Weihai (HITWH 200702) and by the Science and Technology Foundation of Weihai (2007-96).

\section{References (This is "Header 1 " style)}

[1] L. Cheng, J. Gong, X. Yang, C. Fan and P. Han, "Robust affine invariant feature extraction for image matching," IEEE Geoscience and Remote Sensing Letters, Vol. 5, no. 2, pp. 246-250, 2008

[2] H. Neemuchwala, A. Hero, P. Carson, "Image matching using alpha-entropy measures and entropic graphs," Signal Processing, Vol. 85, no. 2 , pp. 277-296, 2005.

[3] Heng-Da Cheng, Manasi Datar, Wen $\mathrm{Ju}$, "Natural scene segmentation based on information fusion and homogeneity property," Proc. Of the Joint Conference on Information Sciences, 2006.

[4] Jin-Wen Tian, Kang Su, Jian Liu, "Image matching based on local entropy difference----algorithm and computer simulation," Journal of Astronautics, Vol. 20, no. 1, pp. 28-32, 1999.

[5] Tong Dong, "Study of reconfigurable storage scheme and multistage interconnection network," Ph.D. Dissertation, Harbin Institute of Technology, pp. 13-77, 1999.

[6] Kai-Kun Dong, Ming-Zeng Hu, BinXing Fang and Zhen-Zhou Ji, "A parallel image processing environment based on image algebra," Journal of computer research and development", Vol. 41, no. 1, pp. 201-206, 2004. 